\title{
Getting to the heart of small RNAs
}

The discovery of small RNA molecules that affect gene expression is one of the watershed developments in molecular biology, but relatively little is known about how these bits of RNA function, especially in higher vertebrates. Now, for the first time in mammals, researchers have identified consequences associated with the deletion of one of these RNA molecules.

MicroRNAs (miRNAs) are short pieces of RNA that do not code for proteins. Complex cellular machinery uses miRNAs to degrade or inhibit the translation of specific sequences of mRNA, preventing the synthesis of corresponding proteins. In early experiments, Eva van Rooij and Eric N. Olson, researchers at the University of Texas-Southwestern Medical Center (Dallas), found that certain miRNAs increased or decreased in cardiac tissue in response to heart stress.

One of these miRNAs, miR-208, is located in an untranslated region of the gene for the alpha myosin heavy chain ( $\alpha \mathrm{MHC}), \alpha \mathrm{MHC}$, along with its cousin $\beta \mathrm{MHC}$, are cardiac proteins responsible for the heart's most characteristic feature: its beat. In the adult human heart, the right ratio of $\beta M H C$ to $\alpha M H C$ is essential for optimum cardiac output, but studies have shown that heart stress throws off that balance by increasing the relative abundance of $\beta M H C$ to $\alpha \mathrm{MHC}$, resulting in poor cardiac performance.

van Rooij and her colleagues decided to knock out the gene for miR-208 in mice to better understand its effects. After deleting the gene, the researchers exposed both mutant and wildtype mice to cardiac stress and found that the mutants did not increase in cardiac $\beta \mathrm{MHC}$ and did not display the characteristic pathological changes seen in the hearts of wildtype mice, evidence that miR-208 regulates $\beta$ MHC expression (Science, doi: 10.1126/science.1139089, published online $22 \mathrm{March}$ ).

Ideally, these findings will be applicable to humans and provide a way to shield people from heart damage- perhaps by selectively suppressing miR-208 expression and

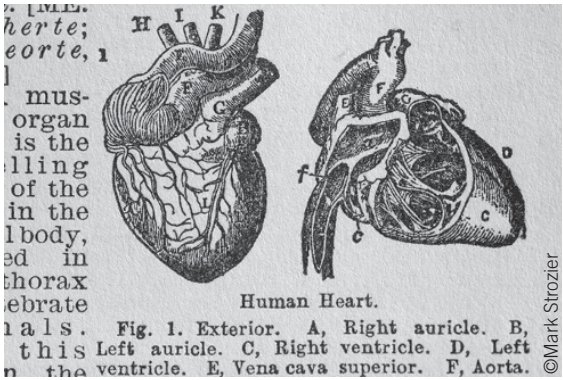

thereby maintaining the ideal $\alpha$ - to $\beta \mathrm{MHC}$ ratio. Still, baseline differences in MHC composition of human and rodent hearts may present obstacles to clinical therapy. "Since the MHC composition differs at baseline in humans [and mice]," van Rooij tells Lab Animal, "it might be very meaningful to determine whether the regulation of the $\beta \mathrm{MHC}$ levels by miR-208 can be recapitulated in larger animal models, like rabbits, in which the baseline $\alpha$ - and $\beta \mathrm{MHC}$ levels are more comparable to the human system."

\section{Owen Young}

\section{COMPLICATED KTNSHIP IN MARMOSETS}

A monkey's uncle may actually be its father, according to a new study showing that germ-cell chimerism is rampant in marmosets. These animals often carry another individual's sperm or egg cells-in this case from their fraternal twin-not only complicating family trees but potentially affecting the parenting practices of these tree-dwelling primates.

Since the 1960 s, scientists have known that marmosets are usually hematopoietic chimeras. Marmoset pregnancies generally produce fraternal twins. During gestation, the two placentas fuse, mingling the blood-producing cells of the developing fetuses. Until now, it was unclear whether this chimerism extended beyond tissues of hematopoietic origin.

While studying parenting and social behavior in the colony of black tufted-eared marmosets (Callithrix kuhlii) at the University of Nebraska (Omaha), Corinna Ross discovered that other tissue types of these animals are often genetic chimeras, including germline tissue. As Ross tells Lab Animal, "Marmosets are well known for having large cooperative breeding groups in which both males and siblings-and sometimes animals that are thought to be unrelated to the breeding pair-help care for infants. In both captivity and in the wild, researchers have noted the formations of not only monogamous pairings, but also polyandrous pairings, in which two males appear to be mating with a breeding female." Ross conducted paternity tests using hair samples from various animals and found that the genetic profile of many of the samples actually matched the sibling of the animal that provided the sample (Proc. Natl. Acad. Sci. USA, doi: 10.1073/ pnas.0607426104, published online 26 March).

Ross, who is now at the University of Texas Health Science Center (San Antonio, TX), went on to genotype samples from 15 tissue types, including liver, lung, heart, and gonads, taken from 36 sets of twins from 15 families. She found some degree of chimerism in all tissue types sampled, including the gonads and sperm, suggesting that the in utero exchange of nonhematopoietic stem cells is common during marmoset development. If some of the sibling's cells take up residence in the gonads, in adulthood the marmoset produces sperm or egg cells containing the genetic material belonging to its twin brother or sister; indeed more than half of the male marmosets sampled produced chimeric sperm.

Wondering how widespread chimerism might affect parenting, Ross tracked the amount of time marmoset parents spent caring for infants. She tells Lab Animal, "We found that males care for chimeric infants more than they care for nonchimeric infants. While we do not know exactly how kin recognition functions in these primates, we are proposing that chimerism may alter a male's attraction to infants because of an altered perception of individuality in these infants."

Tanja Schub 